\title{
Population of high-spin isomeric states following fragmentation of ${ }^{238} \mathbf{U}$
}

M. Bowry, ${ }^{1}$ Zs. Podolyák, ${ }^{1}$ S. Pietri, ${ }^{2}$ J. Kurcewicz, ${ }^{2}$ M. Bunce, ${ }^{1}$ P. H. Regan, ${ }^{1}$ F. Farinon, ${ }^{2}$ H. Geissel, ${ }^{2,3}$ C. Nociforo, ${ }^{2}$ A. Prochazka, ${ }^{2}$ H. Weick, ${ }^{2}$ N. Al-Dahan, ${ }^{1}$ N. Alkhomashi, ${ }^{1}$ P. R. P. Allegro, ${ }^{4}$ J. Benlliure,${ }^{5}$ G. Benzoni, ${ }^{6}$ P. Boutachkov, ${ }^{2}$ A. M. Bruce, ${ }^{7}$ A. M. Denis Bacelar, ${ }^{7}$ G. F. Farrelly, ${ }^{1}$ J. Gerl, ${ }^{2}$ M. Górska, ${ }^{2}$ A. Gottardo, ${ }^{8}$ J. Grębosz, ${ }^{9}$ N. Gregor, ${ }^{2}$ R. Janik, ${ }^{10}$ R. Knöbel, ${ }^{2}$ I. Kojouharov, ${ }^{2}$ T. Kubo, ${ }^{11}$ N. Kurz, ${ }^{2}$ Yu. A. Litvinov, ${ }^{2}$ E. Merchan, ${ }^{2}$ I. Mukha, ${ }^{2}$ F. Naqvi, ${ }^{12}$ B. Pfeiffer, ${ }^{2,3}$ M. Pfützner, ${ }^{13}$ W. Plaß, ${ }^{3}$ M. Pomorski, ${ }^{13}$ B. Riese, ${ }^{2}$ M. V. Ricciardi, ${ }^{2}$ K.-H. Schmidt, ${ }^{2}$ H. Schaffner, ${ }^{2}$ C. Scheidenberger, ${ }^{2,3}$ E. C. Simpson, ${ }^{1}$ B. Sitar,${ }^{10}$ P. Spiller, ${ }^{2}$ J. Stadlmann, ${ }^{2}$ P. Strmen, ${ }^{10}$ B. Sun, ${ }^{2,14}$ I. Tanihata, ${ }^{15}$ S. Terashima, ${ }^{14}$ J. J. Valiente Dobón, ${ }^{8}$ J. S. Winfield, ${ }^{2}$ H.-J. Wollersheim, ${ }^{2}$ and P. J. Woods ${ }^{16}$

${ }^{1}$ Department of Physics, University of Surrey, Guildford GU2 7XH, United Kingdom ${ }^{2}$ GSI, Planckstrasse 1, D-64291 Darmstadt, Germany

${ }^{3}$ IInd Physical Institute, Justus-Liebig University Giessen, D-35392 Giessen, Germany

${ }^{4}$ University of São Paulo, São Paulo 05508-900, Brazil

${ }^{5}$ University Santiago de Compostela, 15706 Santiago de Compostela, Spain

${ }^{6}$ INFN Sezione di Milano, Dipartimento di Fisica, Via Celoria 16, 20133 Milano, Italy

${ }^{7}$ School of Computing Engineering and Mathematics, University of Brighton, Brighton BN2 4GJ, United Kingdom

${ }^{8}$ INFN, Laboratori Nazionali di Legnaro, Legnaro (Padova), Italy

${ }^{9}$ The Henryk Niewodniczański Institute of Nuclear Physics, PL-31-342 Kraków, Poland

${ }^{10}$ Department of Nuclear Physics and Biophysics, Comenius University, Mlynská dolina, 84248 Bratislava, Slovakia

${ }^{11}$ RIKEN Nishina Center, 2-1 Hirosawa, Wako, Saitama 351-0198, Japan

${ }^{12}$ Department of Physics, University of Yale, New Haven, Connecticut 06511-8499, USA

${ }^{13}$ Faculty of Physics, University of Warsaw, PL-00-681 Warsaw, Poland

${ }^{14}$ School of Physics and Nuclear Energy Engineering, Beihang University, Beijing 100191, China

${ }^{15}$ Research Center for Nuclear Physics, 10-1 Mihogaoka, Ibaraki, Osaka 567-0047, Japan

${ }^{16}$ School of Physics and Astronomy, University of Edinburgh, Edinburgh EH9 3JZ, United Kingdom

(Received 2 June 2013; published 16 August 2013)

\begin{abstract}
Isomeric ratios have been determined for 23 metastable states identified in $A \approx 200$ nuclei from $\mathrm{Pt}$ to $\mathrm{Rn}$ near the valley of stability following fragmentation of ${ }^{238} \mathrm{U}$. This includes high-spin states with angular momenta ranging from $(39 / 2) \hbar$ to $25 \hbar$. The experimental results are discussed together with those of similar experiments performed in this mass region. Isomeric ratios are compared with theoretical predictions where the angular momentum of the fragment arises purely due to the angular momentum of nucleons removed from the projectile. The theoretical yield of low-spin states is generally overestimated. In these cases the assumption of $100 \%$ feeding of the isomer may require modification. However, the yield of high-spin isomeric states $\left[I_{m} \geqslant(39 / 2) \hbar\right]$ is significantly underestimated and highlights the requirement for a more complete theoretical framework in relation to the generation of fragment angular momentum. The enhanced population of high-spin states reported here is advantageous to future studies involving isomeric beams at fragmentation facilities such as the Rikagaku Kenkyusho RI Beam Factory (Japan) and next-generation facilities at the Facility for Antiproton and Ion Research (Germany) and Facility for Rare Isotope Beams (USA).
\end{abstract}

DOI: 10.1103/PhysRevC.88.024611

PACS number(s): 23.35.+g, 25.70.Mn, 27.80.+w, 29.30.Kv

\section{INTRODUCTION}

Projectile fragmentation has become a favored tool for producing short-lived exotic nuclear species located far from the valley of stability [1,2]. The production of radioactive ion beams using fragmentation and spallation reactions, in combination with state-of-the-art separator facilities, has driven the most recent discoveries of new isotopes [3]. Techniques such as isotope separation online (ISOL) and in-flight separation are commonly used to isolate the nuclei of experimental interest away from other reaction products. The production and separation of the nuclei of interest for in-flight systems occurs on short time scales $(\lesssim 1 \mu \mathrm{s})$ whereas the extraction of ions from thick ISOL targets can take orders of magnitude longer ( $\gtrsim 1 \mathrm{~ms}$ ) [4]. Consequently, the in-flight method can be used to study short-lived excited isomeric states in exotic nuclei.
Among the first experiments to involve the fragmentation of light ions at energies around $1 \mathrm{GeV} A$ were those performed in the 1970s using the Bevalac accelerator at Lawrence Berkeley Laboratory (USA) [5]. The coupling of high-energy, heavy-ion fragmentation beams (for example, uranium) to powerful $\gamma$ detection arrays is a relatively recent occurrence. For example, the GSI facility in Darmstadt (Germany) possesses the unique capability to provide heavy-ion beams at ultrarelativistic velocities, together with an in-flight fragment separator which was coupled to 105 Euroball cluster detectors (the RISING array). In a stopped-beam configuration, the RISING array (located at the end of the fragment separator until 2009) enabled the detection of delayed $\gamma$ rays following the decay of isomeric states. Such isomer spectroscopy experiments performed with RISING have contributed to our knowledge of the structure of heavy neutron-rich nuclei around ${ }^{208} \mathrm{~Pb}[6,7]$. 
The continued development of the theoretical models underpinning our understanding of fragmentation reactions is essential if the technique is to be used effectively in the future. Certain properties of the fragments such as their production likelihood (cross section), longitudinal and transverse momentum distribution, and angular momentum can all be measured experimentally and therefore used as input to theoretical models. Previous studies have demonstrated (for example, Ref. [8]) that measuring the fraction of nuclei produced in an isomeric state relative to the total number of identified nuclei (isomeric ratio) can provide insight into the generation of angular momentum during peripheral collisions between nuclei at relativistic velocities. In particular, a comparison of experimental isomeric ratios [9] with those predicted by theory revealed that for spins of $17 \hbar$ and $(43 / 2) \hbar$ in ${ }^{214} \mathrm{Ra}$ and ${ }^{215} \mathrm{Ra}$, respectively, the experimental yield was underestimated by a factor $\approx 3-10$.

In this paper, experimental results are presented, exploiting the high-intensity ${ }^{238} \mathrm{U}$ fragmentation beams available at GSI. The results of the current study and that of a parallel work [10] show a similar behavior of the isomeric ratios to that observed in Ref. [9] in heavy nuclei at high spins. In addition, the current study revealed new neutron-rich isotopes [11] and produced new spectroscopic information in some nuclei [12].

\section{EXPERIMENT}

\section{A. Production and selection of radioactive nuclei}

A radioactive beam of heavy ions was produced following fragmentation of a ${ }^{238} \mathrm{U}^{73+}$ primary beam on a $1.63 \mathrm{~g} / \mathrm{cm}^{2}$ beryllium target at $1 \mathrm{GeV}$ per nucleon. The uranium beam was provided by the SIS-18 accelerator at GSI and had an intensity of approximately $2 \times 10^{9}$ particles per spill. Each spill provided (on average) 3-4s of beam on-target, followed by a 2-s beam-off period.

Projectile-like fragments were separated and identified on a particle-by-particle basis by the fragment separator FRS [13], which includes a wedge-shaped energy degrader placed between two sets of dipole magnets. The FRS was used in achromatic mode. A high primary beam energy in combination with a $0.22 \mathrm{~g} / \mathrm{cm}^{2} \mathrm{Nb}$ stripper foil (placed just after the production target) ensured that the majority of the projectilelike fragments entered the FRS fully stripped of electrons [14]. Fragments in the vicinity of ${ }^{205} \mathrm{~Pb}$ were selected according to their magnetic rigidities, $B \rho(\mathrm{Tm})$, proportional to the mass-tocharge ratio, $A / Q$. Additional selectivity in atomic number $Z$ was provided by the energy loss of fragments passing through the degrader. The $A / Q$ of the selected fragments was calculated using time-of-flight and position data from scintillator detectors placed at the intermediate and final focal planes of the separator. The $Z$ value of the fragments was measured using ionization chambers located at the final focal plane. A secondary degrader of appropriate thickness was placed after the ionization chambers at the final focal plane, allowing the radioactive fragments to be slowed down and implanted in a passive ion catcher consisting of a 10-mm-thick Perspex block. The catcher was surrounded by the RISING array $[15,16]$ of 15 high-purity germanium cluster detectors. Delayed $\gamma$ rays emitted following the de-excitation of isomeric states were observed in coincidence with fragments stopped in the catcher. A scintillator was placed after the ion catcher to act as a "veto" for those fragments that did not implant in the catcher.

\section{B. Isomeric ratios}

The isomeric ratio $R_{\exp }$ is defined as the number of nuclei populated in an isomeric excited state relative to the total number of produced nuclei. It was determined by measuring the number of delayed $\gamma$ rays emitted following the deexcitation of an isomeric state relative to the total number of nuclei identified in the FRS as follows:

$$
R_{\exp }=\frac{N_{\gamma}\left(1+\alpha_{T}\right)}{\epsilon b_{t} f_{1}} \times \frac{1}{N_{\text {ion }} f_{2} f_{3} f_{4}},
$$

where $N_{\gamma}$ is the peak area of the delayed $\gamma$ ray and $N_{\text {ion }}$ is the total number of identified nuclei. To obtain the decay yield, $N_{\gamma}$ must be corrected for internal conversion (given by the total conversion coefficient $\alpha_{T}$ ), total branching ratio $b_{t}$, and the detection efficiency $\epsilon$. For some transitions, $\alpha_{T}$ was unavailable. In this case $\alpha_{T}$ was estimated using the online calculator BrIcc [17]. Branching ratios were inferred from $\gamma$-ray transition intensities provided in the literature, corrected for internal conversion. The correction factors $f_{1-4}$ are as follows:

$f_{1}$ : When the fragments are stopped in the catcher, prompt radiation (flash) is detected by some germanium crystals, making them unusable for the detection of delayed $\gamma$ rays. The factor $f_{1}$ is the probability that the RISING detectors are not blinded by the flash and are therefore able to detect delayed $\gamma$ rays. On average, $f_{1}=0.85$.

$f_{2}$ : The average time of flight of the fragments through the FRS is around $300 \mathrm{~ns}$. The factor $f_{2}$ is the fraction of isomeric states that do not decay in-flight and depends on the half-life of the populated isomeric state. If an isomeric state decays mainly through internal conversion the half-life through the separator is effectively increased for fragments fully stripped of electrons.

$f_{3}$ : This factor depends on the width $(\Delta t)$ and position of the time gate applied to the delayed $\gamma$ decay spectrum correlated with a given fragment implanted in the catcher. It takes into account both the finite observation time of the decay of the isomeric state relative to its half-life and the particular point (in time) at which the spectrum is acquired during the decay of the isomeric state.

$f_{4}$ : Secondary nuclear reactions in the degrader at the final focal plane destroy a fraction of the fragments identified in the FRS. The factor $f_{4}$ is the fraction that survive, relative to the total number of fragments entering the degrader. On average, $f_{4}=0.84$.

\section{RESULTS}

\section{A. Identification of reaction residues}

Fragment identification is dependent on the effective separation of different charge states in the secondary beam, which affects the measured $A / Q$ ratio. The energy loss of 


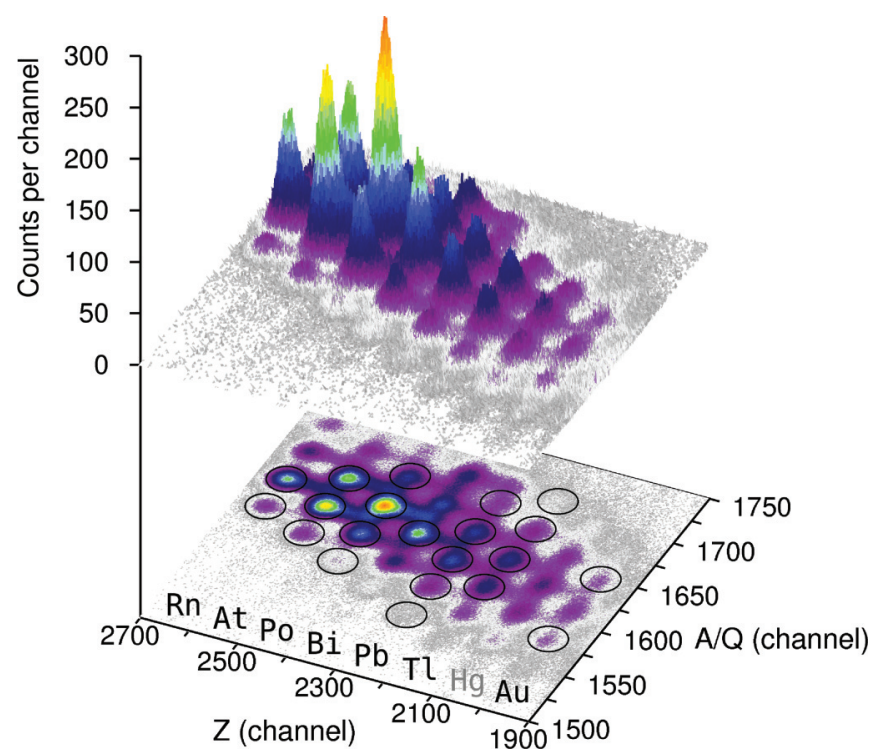

FIG. 1. (Color online) Atomic number $Z$ of radioactive fragments correlated with the mass-to-charge ratio $A / Q$ following projectile fragmentation of uranium. Rings indicate the loci for which delayed $\gamma$ rays were observed in coincidence with nuclei implanted in an ion catcher, identified as ${ }^{214-215} \mathrm{Rn},{ }^{211-213} \mathrm{At},{ }^{208-211} \mathrm{Po},{ }^{207} \mathrm{Bi},{ }^{202-206} \mathrm{~Pb}$, ${ }^{201-204} \mathrm{Tl}$, and ${ }^{195,197} \mathrm{Au}$.

fragments passing through the wedge degrader was used to identify changes in charge state between the intermediate (i.e., before the degrader) and final focal planes of the FRS. The difference in energy loss between two fragments with the same $Z$ is proportional to the difference in $\Delta B \rho$ of each fragment, where $\Delta B \rho$ is the change in $B \rho$ between the intermediate and final focal planes. For example, if two fully stripped fragments with identical $Z$ enter the degrader, one fragment may exit in a hydrogen-like state $\left(+1 e^{-}\right)$while the other remains fully stripped. In this case the hydrogen-like fragment has a lower $\Delta B \rho$ relative to the bare fragment. Therefore a shift in $\triangle B \rho$ observed for fragments with the same $Z$ indicates a change in charge state. To isolate different shifts in charge state, two-dimensional conditions were applied to a plot of degrader energy loss versus energy loss in an ionization chamber (proportional to $Z^{2}$ ) located at the final focal plane. The application of such conditions to the correlated $Z$ versus $A / Q$ data largely negated the interference from charge states in fragment identification [18].

Figure 1 shows a typical particle identification plot in which isotopes of elements from $\operatorname{Pt}(Z=78)$ to $\operatorname{Rn}(Z=86)$ produced in the projectile fragmentation of uranium were unambiguously identified. Other identification plots as well as the number of implanted nuclei for all ions are given in [18]. Delayed $\gamma$ rays were observed in coincidence with those nuclei identified by the solid rings in Fig. 1.

Software gates placed on individual nuclei identified from their $Z$ and $A / Q$ were used to isolate delayed $\gamma$ rays observed in coincidence with fragments implanted in the catcher. In some cases the same nucleus was observed in several identification plots. In this instance, the delayed decay data (i.e., $\gamma$-ray energy and time) associated with a given nucleus

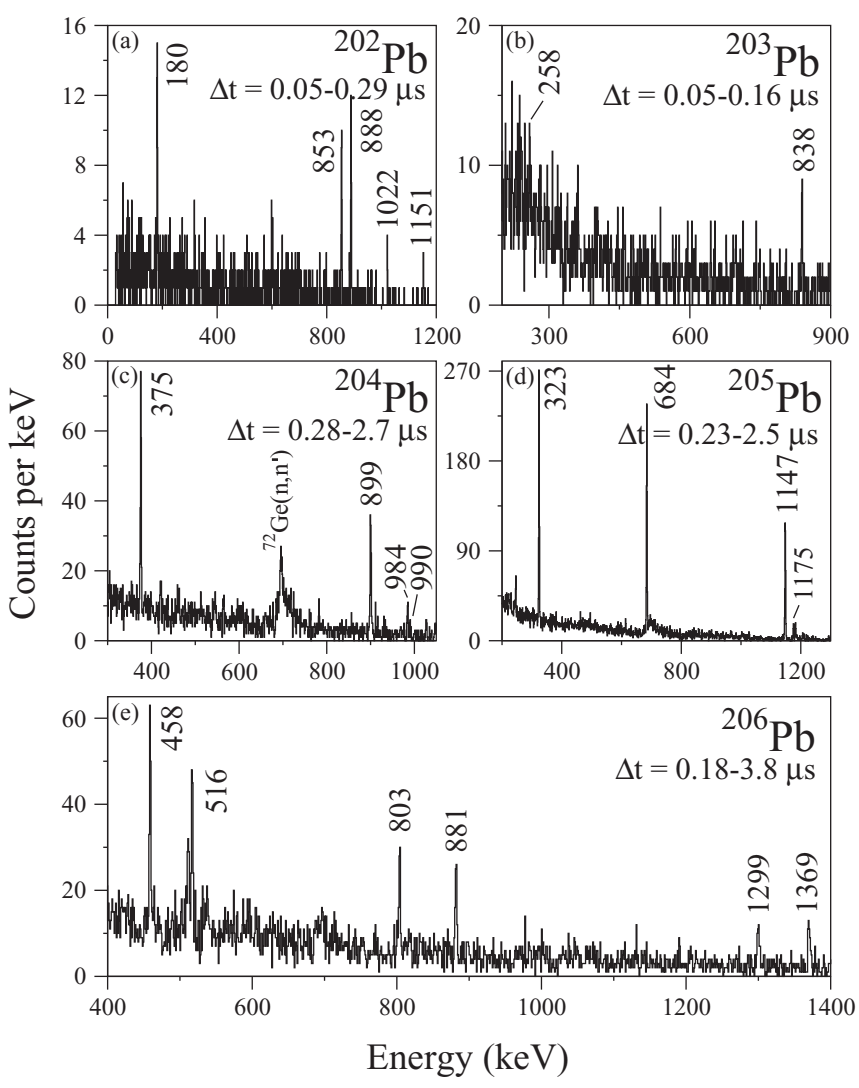

FIG. 2. Delayed $\gamma$-ray spectra associated with ${ }^{202-206} \mathrm{~Pb}$ [(a)-(e)] identified following the de-excitation of known isomeric states [19-23]. A time gate $\Delta t$ was applied to separate the isomeric decay from the prompt radiation flash observed by some germanium detectors following fragment implantation.

in each identification plot were added together to produce the final delayed $\gamma$ spectrum. The delayed decay data were sorted into energy-time matrices and two-dimensional software gates were applied to separate the decay of the populated isomeric state from the prompt radiation flash. Examples of delayed $\gamma$-ray spectra obtained in this way are shown in Fig. 2 for the $\mathrm{Pb}(Z=82)$ isotopic chain. The intensities of transitions observed in these spectra were used in Eq. (1) to determine isomeric ratios (see Sec. III B).

The majority of isomeric states observed here have been previously identified. New spectroscopic information was obtained regarding isomeric states populated in ${ }^{195} \mathrm{Au},{ }^{201} \mathrm{Tl}$, and ${ }^{215} \mathrm{Rn}$ (including half-lives) and were reported in a recent conference proceedings [12].

\section{B. Isomeric ratios}

The isomeric ratios measured in this work are summarized in Table I, which also contains theoretical isomeric ratios. Full details of the experimental isomeric ratios presented in Table I are provided in [18].

In fragmentation reactions, projectile and target nuclei collide at a relative velocity greater than the typical orbital velocities of individual nucleons [24]. Peripheral collisions between nuclei at relativistic velocities have been described 
TABLE I. Summary of experimental isomeric ratios determined in the current work $\left(R_{\exp }\right)$. The spin-parity $\left(I_{m}^{\pi}\right)$, half-life $\left(T_{1 / 2}\right)$, and excitation energy $\left(E^{*}\right)$ of each isomer are taken from the literature, except in the case of ${ }^{195} \mathrm{Au}$, ${ }^{201} \mathrm{Tl}$, and ${ }^{215} \mathrm{Rn}$, where $T_{1 / 2}$ is the one measured in the current work (see Ref. [12]). In cases where the excitation energy is not known precisely, $E^{*}$ is appended with \#. The number of implanted ions $\left(N_{\text {ion }}\right)$ is given after the rejection of events by the veto scintillator. Theoretical isomeric ratios are also shown where $R_{\text {th }}^{f}$ was calculated using a simple analytical formula [Eq. (3)] and $R_{\mathrm{th}}^{\text {stat }}$ was calculated using the Monte Carlo-type model ABRABLA [25]. The uncertainty in $R_{\mathrm{th}}^{\text {stat }}$ is given where appropriate but is otherwise small for the majority of nuclei. $R_{\mathrm{th}}^{\text {stat }}$ for ${ }^{195} \mathrm{Au}$ is the one calculated for $I_{m}^{\pi}=(27 / 2) \hbar$.

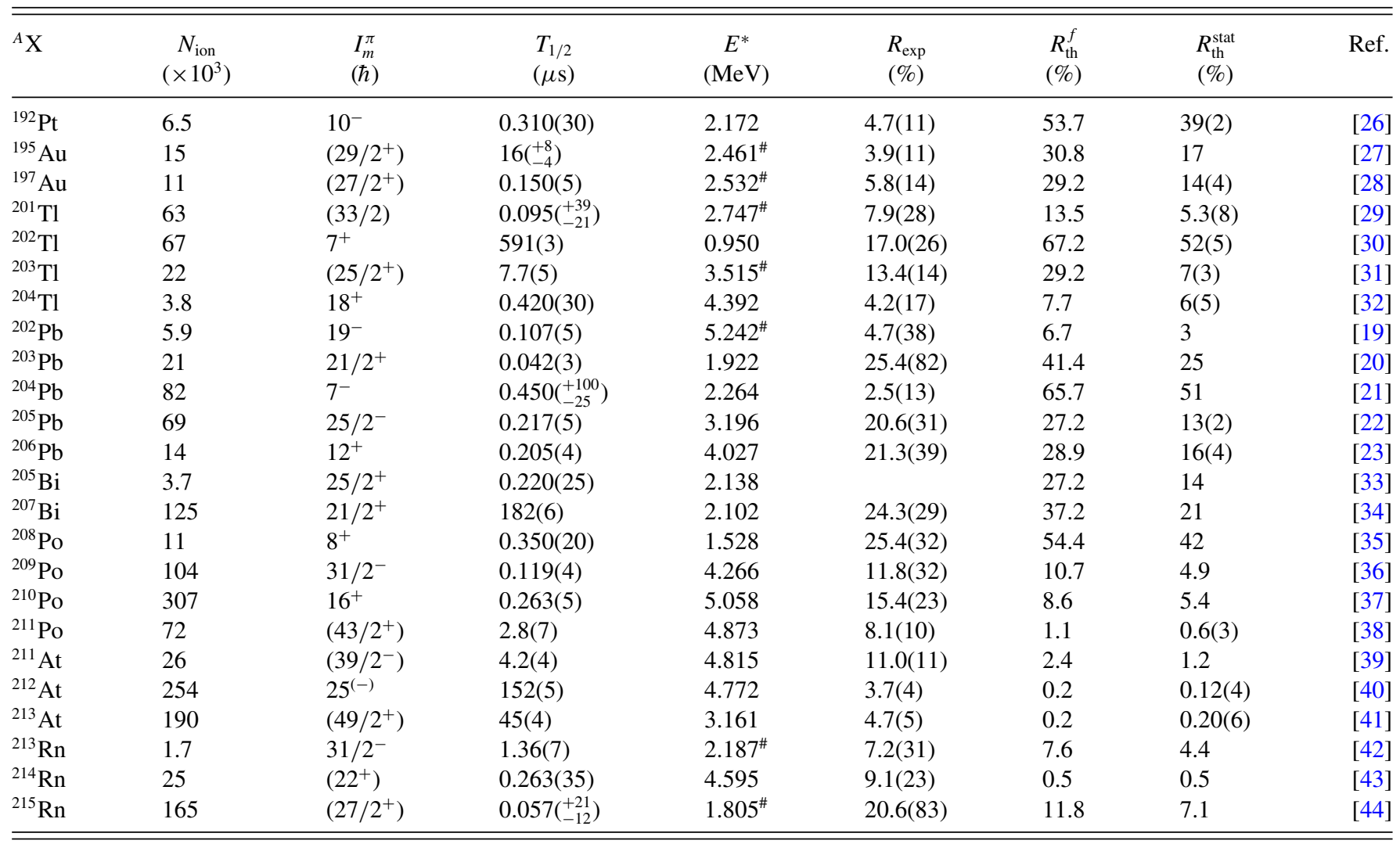

using the two-step "abrasion-ablation" model [24] in which nucleons are removed from the projectile and target nuclei (abrasion), leaving behind highly excited projectile-like and target-like prefragments. The abrasion phase is viewed in terms of the "participant-spectator" system in which the majority of the projectile-target interaction occurs within the participant region of overlap between the colliding nuclei. Prefragments may then evaporate nucleons and other light particles (ablation), losing energy and cooling below the particle separation thresholds.

A simple analytical formula was used to predict the distribution in angular momentum of the projectile-like fragments. The probability $P_{I}$ of populating a given excited state as a function of the total angular momentum $I$ is described as [25]

$$
P_{I}=\frac{2 I+1}{2 \sigma_{f}^{2}} \exp \left(\frac{-I(I+1)}{2 \sigma_{f}^{2}}\right),
$$

where $\sigma_{f}^{2}$ is the spin-cutoff parameter. The theoretical isomeric ratio $R_{\text {th }}^{f}$ is obtained by integrating the probability distribution given by Eq. (2) between the spin of the isomeric state of interest, $I_{m}$, and $I=\infty$ [8]. This is referred to as the sharpcutoff approximation. The assumption is that all excited states populated with $I>I_{m}$ must eventually decay through the isomeric state of interest. This gives

$$
R_{\mathrm{th}}^{f}=\exp \left(\frac{-I_{m}\left(I_{m}+1\right)}{2 \sigma_{f}^{2}}\right) .
$$

Values of $R_{\mathrm{th}}^{f}$ calculated using Eq. (3) are given in column seven of Table I. The spin-cutoff parameter $\sigma_{f}^{2}$, related to the width of the angular momentum distribution, can be calculated based on the formula proposed by Goldhaber [45]:

$$
\sigma_{f}^{2}=\left\langle j_{z}^{2}\right\rangle \frac{\left(A_{p}-A_{f}\right)\left(v A_{p}+A_{f}\right)}{(v+1)^{2}\left(A_{p}-1\right)},
$$

where $A_{p}$ is the mass of the projectile, $A_{f}$ is the mass of the final fragment, and $\left\langle j_{z}^{2}\right\rangle$ is the average square angular momentum projection of the nucleons removed from the projectile. The parameter $v$ is the average number of nucleons evaporated per nucleon removed from the projectile during the collision. Here $v=2$ [25], if one assumes that each evaporated nucleon removes around $13 \mathrm{MeV}$ of excitation energy from the prefragment, or around half the average excitation energy induced per abraded nucleon (27 MeV [46]). The average square angular momentum projection of nucleons varies 
smoothly with the mass of the nucleus for large excitation energies around $100 \mathrm{MeV}$ [47]:

$$
\left\langle j_{z}^{2}\right\rangle=k A_{p}^{\frac{2}{3}}\left(1-\frac{2 \beta}{3}\right),
$$

provided that a sufficient number of nucleons are removed from the projectile $(\Delta A \gtrsim 10)$. The parameter $\beta$ is the quadrupole deformation parameter (and here $\beta$ is assumed to equal zero). In the current study, as in Ref. [25], we assume the scale factor $k$ equals 0.16 . The factor $k$ varies depending on the type of nuclear potential used to calculate $\left\langle j_{z}^{2}\right\rangle$.

Column eight of Table I contains theoretical isomeric ratios calculated with the Monte Carlo-type model ABRABLA described by de Jong et al. [25] $\left(R_{\mathrm{th}}^{\text {stat }}\right)$. In the ABRABLA model the spin-cutoff parameter is partly derived from the nuclear equations of state for a Fermi gas [25]. The prefragment excitation energy is calculated via Monte Carlo methods for a given number of abraded nucleons. The sharpcutoff approximation was also applied to the ABRABLA calculations.

\section{DISCUSSION}

In Table II details of previous experimental isomeric ratio studies at GSI using ${ }^{208} \mathrm{~Pb}$ and ${ }^{238} \mathrm{U}$ fragmentation beams are summarized. Table II includes relevant experimental parameters, details of the fragments analyzed $(Z, A)$, and the range (in angular momentum) of the measured isomeric ratios. The last line details the current study for comparison.

The table shows that a wide range of isomeric states with angular momentum ranging from $(5 / 2) \hbar$ to $(55 / 2) \hbar$ have been studied. The highest-spin state for which an isomeric ratio has been measured in this mass region is the $I_{m}^{\pi}=\left(55 / 2^{+}\right)$state in

${ }^{213} \mathrm{Rn}$ [42], obtained in the study by Bacelar et al. [10]. Table II also shows the number of nuclei identified (from characteristic delayed $\gamma$ rays) and the total number of isomeric ratios measured in each work. In Ref. [10] the number of isomeric ratios presented is significantly greater than the number of identified nuclei. This is due to the yield of several isomeric states having been measured in the same nucleus, for example, the sequence of states with $I_{m}^{\pi}=\left(25 / 2^{+}\right),\left(31 / 2^{-}\right),\left(43 / 2^{-}\right)$, and $\left(55 / 2^{+}\right)$in ${ }^{213} \mathrm{Rn}$ [42]. In the current study 23 isomeric ratios were measured out of a total of 24 nuclei identified from delayed $\gamma$ rays. The isomeric ratios of the $25 / 2^{+}$and $21 / 2^{+}$ isomeric states in ${ }^{205} \mathrm{Bi}$ [33] could not be reliably determined. This was due to their similar lifetimes [33] and small separation in excitation energy, where the low-energy transition directly de-exciting the $25 / 2^{+}$state (decaying to the $21 / 2^{+}$state) has an energy similar to that of Bi x-ray lines.

Data from the literature summarized in Table II are plotted in Fig. 3, which shows the location $(N, Z)$ of nuclei in which isomeric ratios have been measured, including the current study. Studies performed at GSI using uranium fragmentation beams have focused predominantly on neutron-deficient nuclei from $\mathrm{Hg}$ to Ac, "northwest" of the valley of stability near the $Z=82$ and $N=126$ major shell closures. The concentration of measurements in this area of the nuclear chart is perhaps due to the good availability of isomeric states with lifetimes of the order of microseconds, including many high-spin states, in nuclei near doubly-magic ${ }^{208} \mathrm{~Pb}$ (e.g., ${ }^{211-213} \mathrm{At}$ ). Studies using lead fragmentation beams have measured isomeric ratios in neutron-deficient nuclei between $\mathrm{Sm}$ and $\mathrm{Ho}[8,51]$ and in mid-shell nuclei between $\mathrm{Hf}$ and Re [8]. Isomeric ratios were also measured in many neutron-rich isotopes southwest of ${ }^{208} \mathrm{~Pb}$ in the nuclear chart from $\mathrm{Ta}$ to $\mathrm{Tl}[6,49]$.

\section{A. Analysis of isomeric ratios}

In Fig. 4 experimental isomeric ratios are plotted as a function of angular momentum and excitation energy of the identified isomeric state. The top and bottom panels of Fig. 4 show results obtained using uranium and lead projectiles, respectively. In general, the experimental isomeric ratios decrease with increasing angular momentum. The trend appears more noticeable for the uranium data than for lead, where the latter contain a lower fraction of measurements performed at high angular momentum. However, the handful of isomeric ratios measured for high-spin states with $I_{m} \geqslant 19 \hbar$ [51] appear to show a similar decrease toward high angular momentum. The observed reduction in $R_{\exp }$ is consistent with the expected decrease in the theoretical isomeric ratio $R_{\mathrm{th}}^{f}$ as a function of increasing angular momentum, although the data are associated with a range of different projectile-target mass losses.

TABLE II. Details of experimental measurements of isomeric ratios in heavy nuclei. The number of nuclei identified from characteristic delayed $\gamma$ rays and isomeric ratios (IR) measured in each study are shown in columns eight and nine, respectively. Studies listed here were performed at GSI using either ${ }^{208} \mathrm{~Pb}$ or ${ }^{238} \mathrm{U}$ fragmentation beams.

\begin{tabular}{|c|c|c|c|c|c|c|c|c|}
\hline Study [Ref.] & Projectile & $E(\mathrm{GeV} A)$ & Target $(\mathrm{Be})\left(\mathrm{g} / \mathrm{cm}^{2}\right)$ & $Z$ & $A$ & $I_{m}(\hbar)$ & Nuclei & IR \\
\hline Pfützner et al. [1] & ${ }^{238} \mathrm{U}$ & 1 & 1.0 & $81 \rightarrow 83$ & $203 \rightarrow 212$ & $7 \rightarrow 20$ & 10 & 10 \\
\hline Pfützner et al. [8] & ${ }^{208} \mathrm{~Pb}$ & 1 & 1.6 & $62 \rightarrow 80$ & $136 \rightarrow 206$ & $5 / 2 \rightarrow 35 / 2$ & 18 & 26 \\
\hline Caamaño et al. [49] & ${ }^{208} \mathrm{~Pb}$ & 1 & 1.6 & $73 \rightarrow 79$ & $188 \rightarrow 203$ & $7 \rightarrow 19 / 2$ & 11 & 13 \\
\hline Podolyák et al. $[9,50]$ & ${ }^{238} \mathrm{U}$ & 0.9 & 1.0 & $86 \rightarrow 89$ & $207 \rightarrow 215$ & $13 / 2 \rightarrow 43 / 2$ & 13 & 13 \\
\hline Bacelar et al. [10] & ${ }^{238} \mathrm{U}$ & 1 & 2.5 & $84 \rightarrow 89$ & $198 \rightarrow 215$ & $13 / 2 \rightarrow 55 / 2$ & 24 & 41 \\
\hline Myalski et al. [51] & ${ }^{208} \mathrm{~Pb}$ & 1 & 2.5 & $62 \rightarrow 67$ & $142 \rightarrow 153$ & $11 / 2 \rightarrow 27$ & 9 & 10 \\
\hline This work & ${ }^{238} \mathrm{U}$ & 1 & 1.6 & $78 \rightarrow 86$ & $192 \rightarrow 215$ & $7 \rightarrow 25$ & 24 & 23 \\
\hline
\end{tabular}




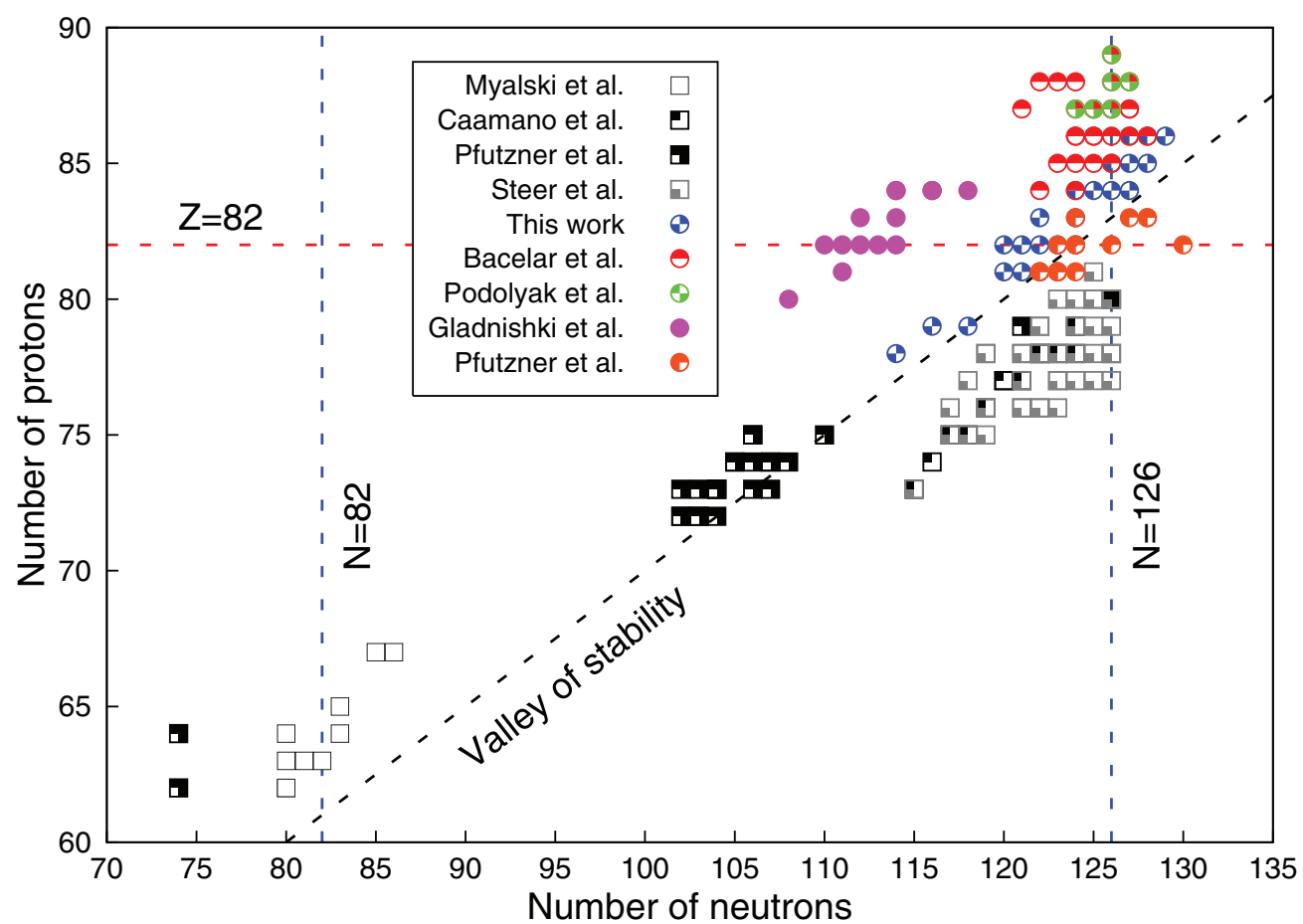

FIG. 3. (Color online) Nuclear chart ( $N$ vs $Z$ ) summarizing selected experimentally measured isomeric ratios in heavy nuclei. Squares indicate nuclei produced using ${ }^{208} \mathrm{~Pb}$ fragmentation beams $[6,8,49,51]$ while circles show nuclei produced using ${ }^{238} \mathrm{U}[1,9,10,48,50]$, including the current work. See Table II for details.
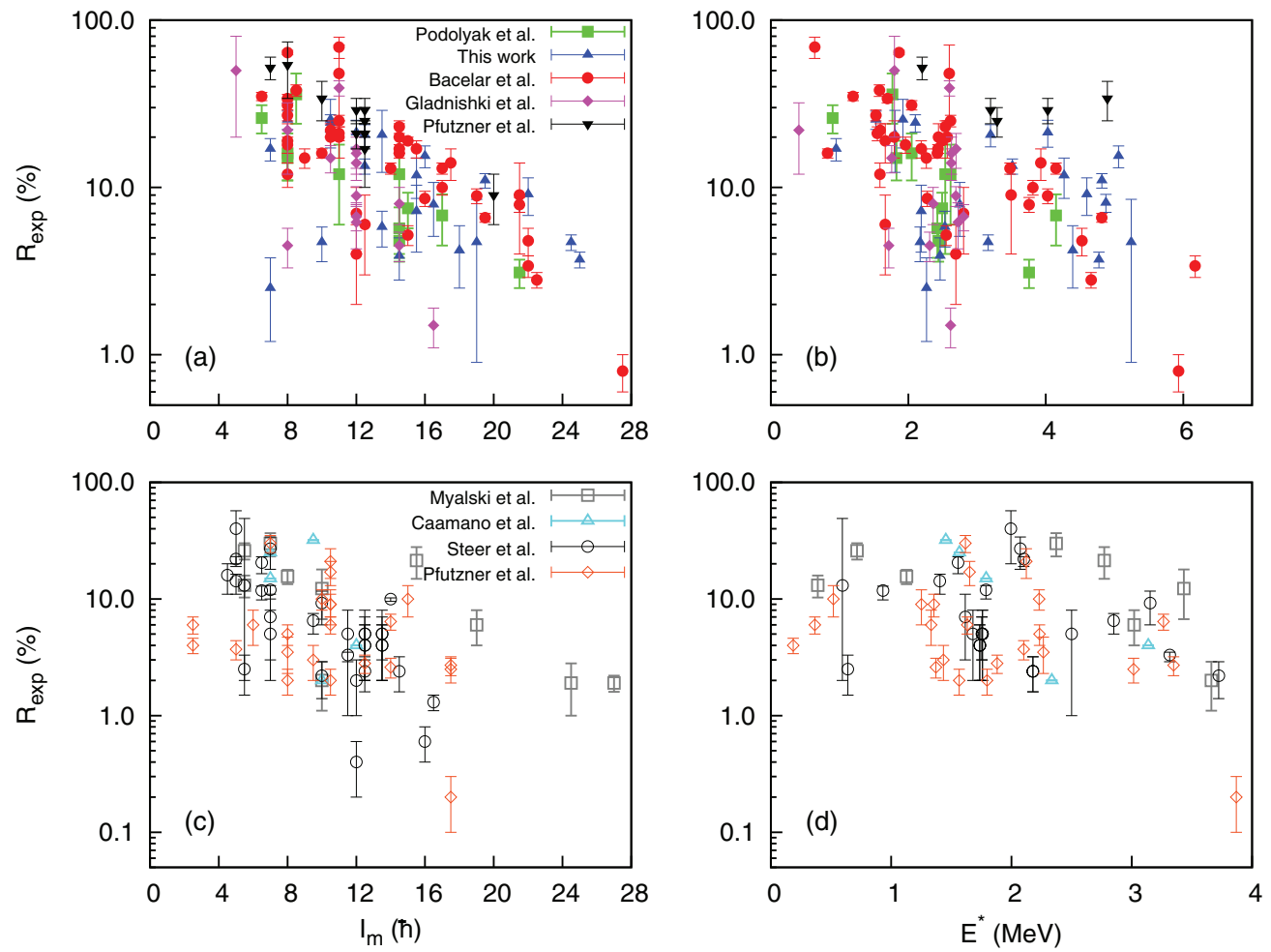

FIG. 4. (Color online) Experimental isomeric ratios plotted as a function of angular momentum and excitation energy of the isomeric state for both ${ }^{238} \mathrm{U}[(\mathrm{a})-(\mathrm{b})]$ and ${ }^{208} \mathrm{~Pb}[(\mathrm{c})-(\mathrm{d})]$ fragmentation studies [1,6,8-10,48-51]. See Table II for details. 
$\Delta \mathrm{A}(\mathrm{u})$

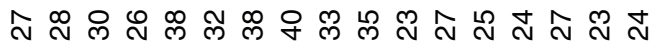

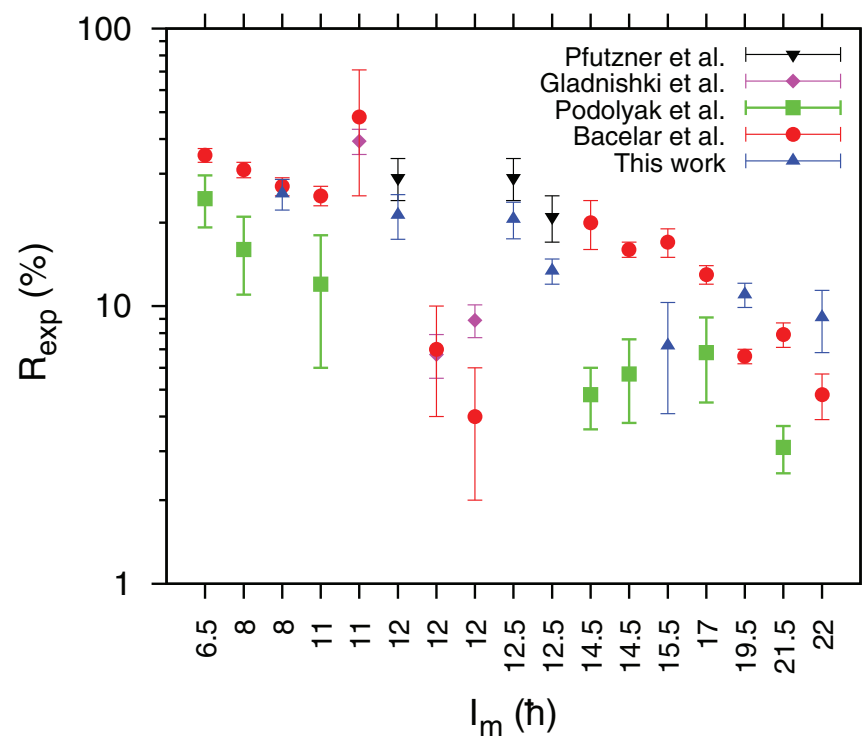

FIG. 5. (Color online) Comparison of experimental isomeric ratios reported in ${ }^{238} \mathrm{U}$ fragmentation studies $[1,9,10,48,50] . R_{\exp }$ is plotted against the angular momentum of the isomeric state for identical states in the same nucleus. The mass loss of the fragments compared with the beam is also shown. See Table II for details.

Figure 4(b) shows that there is also a decrease in the experimental isomeric ratio as a function of increasing excitation energy of the isomeric state, although the correlation appears weaker than in the case of increasing angular momentum and is only noticeable for the uranium data. However, this is consistent with the expected behavior of the relative isomeric yields if one assumes that along the yrast line, higher spin states (for which the predicted yields are lower) are associated with higher excitation energies. The data for $R_{\exp }$ as a function of excitation energy following lead fragmentation are largely flat up to $4 \mathrm{MeV}$ [Fig. 4(d)], not including the observation of two high-spin, high-energy isomeric states in ${ }^{147} \mathrm{Gd}$ and ${ }^{148} \mathrm{~Tb}[51]\left[I_{m}^{\pi}=\left(49 / 2^{+}\right)\right.$and $\left(27^{+}\right)$, respectively] at an excitation energy of around $8.6 \mathrm{MeV}$ where $R_{\exp } \approx 2 \%$ in both cases.

In Fig. 5 the reproducibility of experimentally measured isomeric ratios following uranium fragmentation is examined. $R_{\text {exp }}$ is plotted against the angular momentum of the isomeric state of interest and includes measurements taken from the current work and from Refs. [1,9,10,48]. The mass loss $\Delta A$ of the fragments compared with the beam is shown on the secondary $x$ axis. Each comparison indicates where the isomeric ratio has been measured for identical isomeric states identified in the same nucleus. The largest difference observed between two experimental data points is for the $\left(29 / 2^{+}\right)$state in ${ }^{215} \mathrm{Ac}$ [52] $(\Delta A=23)$, where the isomeric ratio measured in Ref. [10] is just over a factor of 4 greater than the value of $R_{\text {exp }}$ presented in Ref. [9] (and neither of these measurements agree within uncertainty limits).

Good agreement (less than a factor of 2) is achieved for isomeric ratios measured in the current study and in
Ref. [1] for the $12^{+}$state in ${ }^{206} \mathrm{~Pb}$ [53] $(\Delta A=32)$, the $25 / 2^{-}$state in ${ }^{205} \mathrm{~Pb}[22](\Delta A=33)$, and the $\left(25 / 2^{+}\right)$state in ${ }^{203} \mathrm{Tl}$ [31] $(\Delta A=35)$. Isomeric ratios measured in the current work and in Ref. [1] for ${ }^{205-206} \mathrm{~Pb}$ also agree within the uncertainty limits. Similarly good agreement is obtained when comparing the majority of the current results with those of the parallel study [10], particularly for the $8^{+}$state in ${ }^{208}$ Po [35] $(\Delta A=30)$, where the measurements lie within the uncertainty limits.

In Fig. 5, isomeric ratios determined in Ref. [9] are consistently lower than those in Ref. [10] (square and circular data points, respectively). This may indicate some systematic dependence of the isomeric ratios, possibly due to different experimental parameters. For example, the primary beam velocity (see Table II) can greatly affect the number of fragments in a particular charge state. At higher velocities, a greater number of fragments enter the fragment separator as fully stripped ions [14]. A greater number of ionic charge states could contribute to the complexity of identification of the reaction residues and affect the measured isomeric ratios.

The effect of the longitudinal momentum of the fragments upon the isomeric ratio must also be considered. This was shown experimentally by Daugas et al. [54] using a ${ }^{92} \mathrm{Mo}$ fragmentation beam at intermediate energies, where a strong dependence of the isomeric ratio upon the longitudinal momentum distribution of the fragments was observedparticularly for fragments close to the mass of the projectile. In addition, recent analysis [55] of two-proton removal reactions populating $5^{-}$and $10^{+}$isomeric states in ${ }^{206} \mathrm{Hg}$ (following reactions of a relativistic ${ }^{208} \mathrm{~Pb}$ beam upon a beryllium target) has demonstrated the dependence of the isomeric ratio upon the fragment momentum, which appears enhanced toward the extremes of the distribution. However, for targets of a similar thickness to those used in this work and several previous studies (around $2 \mathrm{~g} / \mathrm{cm}^{2}$; see Table II) it was concluded that the dependence of the isomeric ratio on the momentum acceptance of the separator is small [55]. It is also expected that for larger mass loss between the projectile and target nuclei (such as that described in the abrasion-ablation model), the dependence on momentum acceptance is further reduced. Therefore it is expected that the longitudinal momentum selection does not play a significant role in the studies discussed here.

\section{B. Comparison with theory}

Figure 6 shows the experimental isomeric ratios from the current work (Table I) compared with the theoretically determined isomeric ratios as a function of angular momentum. The ratio $R_{\text {exp }} / R_{\text {th }}$ compares the experimental results with the prediction of Eq. (3) $\left(R_{\mathrm{th}}^{f}\right.$ : filled circles) and the Monte Carlo-type model ABRABLA ( $R_{\mathrm{th}}^{\text {stat: }}$ open circles). The inset in Fig. 6 shows the ratio $R_{\mathrm{th}}^{f} / R_{\mathrm{th}}^{\text {stat }}$ plotted as a function of angular momentum. $R_{\mathrm{th}}^{f}$ has no associated error bar whereas statistical uncertainties apply to $R_{\mathrm{th}}^{\text {stat }}$ (see Table I). In this mass region, the ABRABLA model consistently predicts lower isomeric ratios than those calculated analytically. As a consequence the ratio $R_{\exp } / R_{\mathrm{th}}$ is usually higher when comparing to the ABRABLA data. Figure 7 compares the 


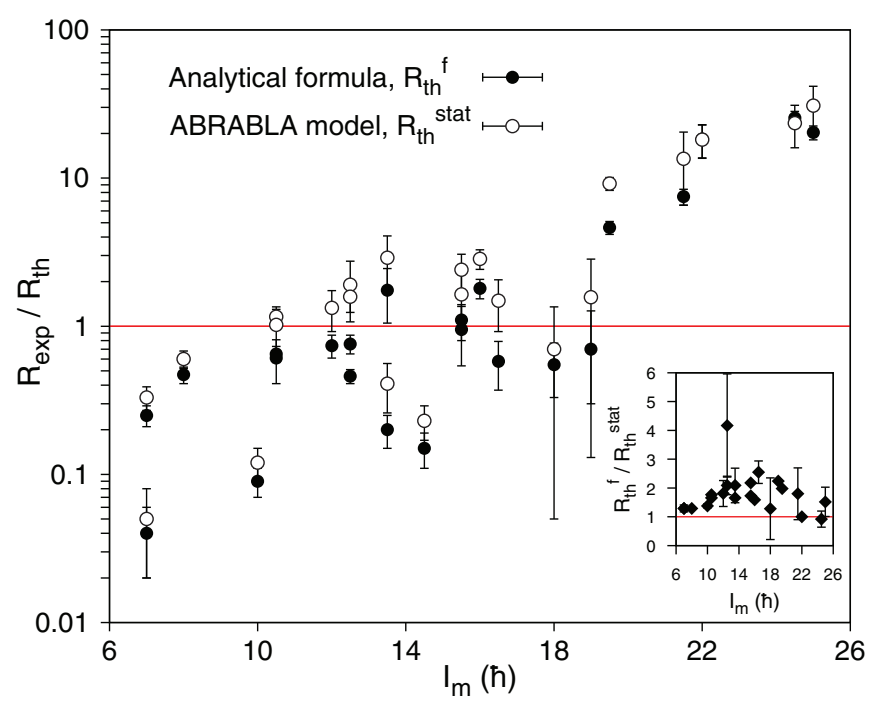

FIG. 6. (Color online) Experimental isomeric ratios determined in the current study (see Table I) compared with the theoretical population plotted as a function of angular momentum of the isomeric state. Inset: $R_{\mathrm{th}}^{f} / R_{\mathrm{th}}^{\mathrm{stat}}$. The theoretical isomeric ratio calculated using Eq. (3) is compared with the yield provided by the Monte Carlo-type model ABRABLA as a function of angular momentum.

experimental and theoretical isomeric ratios as a function of angular momentum but includes results from other uranium fragmentation studies $[1,9,10,48,50]$ as well as the current work.

\section{Lower-spin isomeric states}

The sharp-cutoff approximation is assumed to be a good model for states lying on or close to the yrast line as these states are populated during the final phase of de-excitation of the fragment. Once the yrast line is reached, further de-excitation is most likely to continue along this path where (typically) only a small fraction of the decay intensity is diverted to nonyrast states. For this reason yrast isomeric states should be well populated.

The majority of the isomeric states identified in the current study can be described as yrast based on the available spectroscopic data, although possible exceptions occur where the exact spin and/or excitation energy is unknown (see Table I). In Figs. 6 and 7 the majority of isomeric ratios measured for $I_{m} \lesssim 18 \hbar$ fall either below or just above $R_{\text {exp }} / R_{\mathrm{th}}=1$. For $R_{\mathrm{exp}} / R_{\mathrm{th}}$ ratios less than 1 , the theoretical calculations overestimate the isomeric ratio when compared with the experimental data. The relatively poor isomeric yields measured for particular yrast isomers [for example, the $7^{-}$state in ${ }^{204} \mathrm{~Pb}$ [21] where $\left.R_{\exp } / R_{\mathrm{th}}^{f}=0.04(2)\right]$ appear contrary to the assumption of the sharp-cutoff approximation that yrast isomers are well populated. However, we know that the sharp-cutoff approximation is not always valid, and when sufficient information is available regarding excited states, one can correct for it.

Given sufficient spectroscopic information regarding the feeding of the populated isomeric state, it is useful to consider a correction factor $\varphi[56]$ which can be applied to the theoretical
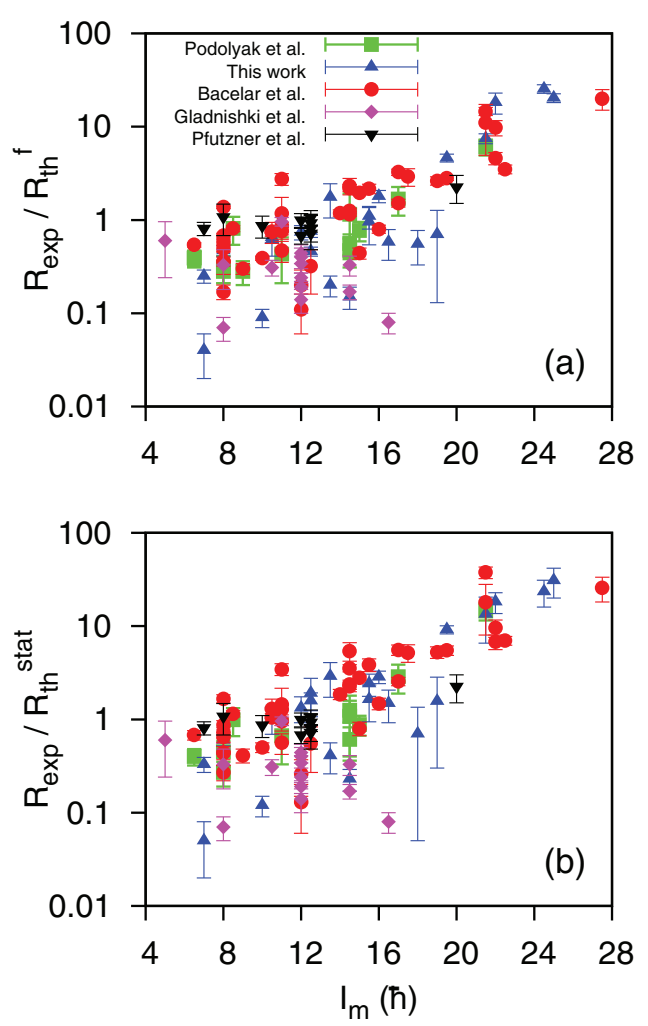

FIG. 7. (Color online) Experimental isomeric ratios measured following uranium fragmentation $[1,9,10,48,50]$ compared with the theoretical population plotted as a function of angular momentum of the isomeric state, including results from the current work. Data in the top and bottom panels are compared with isomeric yields calculated using (a) Eq. (3) and (b) the Monte Carlo-type model ABRABLA [25], respectively. See Table II for details.

isomeric ratio. The corrected theoretical isomeric ratio is $R_{\mathrm{th}}^{\text {corr }}=R_{\mathrm{th}} \varphi$. A correction can be calculated by dividing the total intensity $(\gamma+$ internal conversion) of transitions feeding the isomeric state by the total intensity of transitions both feeding and bypassing the state. In the limit of $100 \%$ feeding of the isomer, $\varphi$ is equal to unity. Correction factors were calculated for isomeric states identified in ${ }^{204} \mathrm{Tl},{ }^{208} \mathrm{Po},{ }^{211} \mathrm{At}$, and ${ }^{213} \mathrm{Rn}$. Spectroscopic data from nuclear reactions involving heavy projectiles were chosen to represent as best as possible the range of feeding and bypassing transitions close to the populated isomers. For each of these nuclei the estimated value of $\varphi$ was $\geqslant 0.9$, leading to a slight decrease in the theoretical isomeric ratio. This in turn causes a small upward shift in the ratio $R_{\mathrm{exp}} / R_{\mathrm{th}}$ when substituting $R_{\mathrm{th}}$ for $R_{\mathrm{th}}^{\text {corr }}$. However, the overall effect of such corrections is small. Note that the experimental data are compared to uncorrected values of $R_{\text {th }}^{f}$ and $R_{\mathrm{th}}^{\text {stat }}$ in Figs. 6 and 7.

\section{High-spin isomeric states}

Prior to the current work and the parallel work of [10], only two experimental isomeric ratios were measured for high-spin states $\left(I_{m} \gtrsim 17 \hbar\right)$ in heavy nuclei around $A \approx 200$ [9] using a uranium fragmentation beam. In Fig. 6, the ratios $R_{\exp } / R_{\mathrm{th}}$ for high-spin isomeric states observed in ${ }^{211} \mathrm{At},{ }^{211} \mathrm{Po},{ }^{214} \mathrm{Rn}$, 
${ }^{213} \mathrm{At}$, and ${ }^{212} \mathrm{At}$ [in ascending order of angular momentum from $(39 / 2) \hbar$ to $25 \hbar$ ] are all greater than unity, indicating that the experimental yields are enhanced relative to theoretical predictions. Moreover, the discrepancy between experiment and theory grows with increasing angular momentum up to a factor of around 30 for the highest-spin state. The same general trend is observed when comparing experimental data to either the analytically or statistically calculated populations (filled and open circles, respectively). It is important to note that any corrections made to the sharp-cutoff approximation in this region of high angular momentum only serve to increase the discrepancy between experiment and theory.

The greater-than-expected population of high-spin isomeric states reported here is consistent with the results of similar studies using uranium fragmentation beams [9,10], displayed in Fig. 7. The discrepancies between the experimental isomeric ratios (Fig. 5) do not affect this general conclusion. The same effect is not observed in the fragmentation of ${ }^{208} \mathrm{~Pb}$ beams. However, this is because the high-spin isomers studied [51] occur in nuclei with masses very different from that of the beam.

\section{Contributions to the final fragment angular momentum}

Estimates of the isomeric ratios obtained using the sharpcutoff approximation are upper limits and therefore cannot provide any additional population to reconcile the experimental and theoretical data at high angular momentum. Both the ABRABLA model and its analytical counterpart consider angular momentum production only in the abrasion phase of the reaction [25] such that the angular momentum of the final fragment depends only on the removal of nucleons from the single-particle orbits of the projectile.

A hybrid model incorporating the effect of both abrasion and ablation upon the fragment angular momentum has been developed [57] in which the evaporation of light particles from the projectile-like prefragment was observed to increase the average angular momentum by approximately $60 \%$ [57]. The predicted isomeric yields compare reasonably well for several isomers with $I_{m} \approx 15 \hbar$ but still underestimate the experimentally measured population of high-spin states observed in ${ }^{214-215} \mathrm{Ra}\left(17^{-}\right.$and $43 / 2^{-}$, respectively [9]) by a factor $\approx 2-4$ [57]. The effect of particle-hole spin coupling (i.e., excited holes and particles near the Fermi surface of the projectile prefragment) upon the angular momentum was also considered in [57], albeit not explicitly but rather with its effect being simulated by multiplying the spin-cutoff parameter by a factor of 2 . This was shown to adequately reproduce the measured population of the $43 / 2^{-}$state in ${ }^{215} \mathrm{Ra}$. A similar exercise has been performed for the results of the current study. In Fig. 8, the experimental isomeric ratios are compared with those predicted using the analytical formula where $\sigma_{f}^{2}$ [Eq. (4)] was multiplied by 2 . The overall effect upon the data is to cap the ratio $R_{\text {exp }} / R_{\text {th }}$ at around 1 . The measured isomeric yield of the highest-spin states $\left(I_{m} \geqslant 39 / 2 \hbar\right)$ now appears to agree reasonably well with the prediction of the analytical formula, whereas that of the lower-spin states simply appears shifted below $R_{\exp } / R_{\text {th }}=1$, with little change in the relative positions of the measurements.

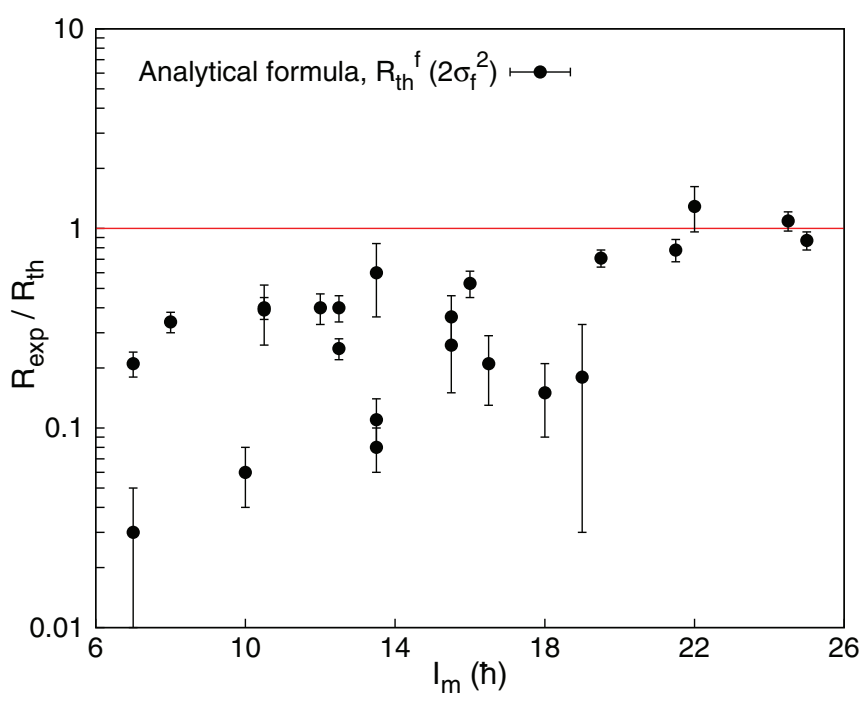

FIG. 8. (Color online) Isomeric ratios determined in the current study (see Table I) compared with the theoretical population predicted by the analytical formula only [Eq. (3)] plotted as a function of angular momentum of the isomeric state. The spin-cutoff parameter in Eq. (3) was multiplied by a factor of 2 .

It has been suggested that projectile-target "friction" $[9,58]$ may contribute to the angular momentum of the fragments. This effect was originally observed during the fragmentation of light projectiles $\left({ }^{12} \mathrm{C},{ }^{16} \mathrm{O}\right)$ [59] where the fragment velocities were degraded relative to that of the beam. A similar effect has since been observed for heavier beams including uranium $[60,61]$. The frictional force that tugs on the projectile during the collision (slowing it down) originates from the energy required to remove nucleons from the Fermi surface (binding energy). Existing theories of angular momentum generation following the relativistic fragmentation of heavy ions cannot adequately reproduce the population of high-spin isomeric states. The source of this discordance may be understood if contributions to the fragment angular momentum, including projectile-target friction and the removal of nucleons through abrasion and ablation, can be better quantified.

\section{CONCLUSIONS}

Experimental isomeric ratios have been determined for 23 metastable states identified in $A \approx 200$ nuclei from $\mathrm{Pt}$ to $\mathrm{Rn}$, populated following the projectile fragmentation of a uranium beam with $E=1 \mathrm{GeV} A$. Results were compared with previously measured isomeric ratios in heavy nuclei produced in fragmentation of both uranium and lead beams. In general the experimental isomeric ratios are shown to decrease as a function of increasing angular momentum and excitation energy of the isomeric state of interest. For lead beams, the experimental isomeric ratios remain fairly constant (around $10 \%$ ) for excitation energies below $4 \mathrm{MeV}$.

The data were compared with theoretical predictions provided by a state-of-the-art Monte Carlo-type model ABRABLA [25] and by a simple analytical formula, under the assumption of $100 \%$ feeding of the populated isomer. 
The experimental yields of high-spin isomeric states, which probe the tail of the fragment spin distribution, are consistently underpredicted by theory by a factor $\approx 5-30$ for $I_{m} \geqslant(39 / 2) \hbar$; the discrepancy increases with increasing angular momentum. The population deficit is interpreted as a limitation of the theoretical models in assuming a purely single-particle contribution to the fragment angular momentum. The experimental and theoretical yields of high-spin states were partially reconciled via an empirical method using a larger-width spin distribution $\left(2 \sigma_{f}^{2}\right)$. However, further investigation is required to attribute an appropriate angular momentum generating mechanism (e.g., ablation or projectile-target friction) to any artificial enhancement of the spin distribution. Isomeric ratios measured for $I_{m} \lesssim 18 \hbar$ are in some instances well reproduced by theory although the population of states below about $10 \hbar$ are all overestimated. In cases where the isomeric ratio is significantly lower than predicted it is likely that the assumption of $100 \%$ feeding of the populated isomer may be invalid, even for yrast isomers. Nuclear structure information specific to each isomeric state is the key to understanding this discrepancy.

Given the greater-than-expected population of high-spin isomeric states reported here, an opportunity arises to exploit the increased isomeric beam yield in future experiments. For example, studies performed using the experimental storage ring ESR at GSI complement spectroscopic data obtained through the observation of delayed $\gamma$ rays where the excitation energy of the populated isomer(s) can be determined directly [62,63]. A high isomeric yield may also facilitate studies involving secondary nuclear reactions (such as Coulomb excitation or nucleon knockout) of radioactive fragments populated in excited isomeric states [64]. Information on highspin excited states could be extracted that would otherwise be inaccessible.

New experimental information obtained here regarding the isomeric ratios of high-spin states provides improved constraints for theoretical studies and may help in the development of more accurate models of projectile fragmentation in the relativistic regime. Moreover, with the development of next-generation high-intensity radioactive ion beam facilities and high-efficiency detector arrays, the resulting increase in experimental sensitivity will allow high-precision measurements of isomeric ratios over a greater range of exotic nuclei.

\section{ACKNOWLEDGMENTS}

The expert assistance of the GSI technical staff in operating the accelerators and the FRS is gratefully acknowledged. The work is supported by the UK Science and Technologies Facilities Council (STFC). B.S. is supported by the NCET under Grant No. NCET-09-0031 and NSFC under Grants No. 11035007 and No. 11105010.
[1] M. Pfützner et al., Phys. Lett. B 444, 32 (1998).

[2] Z. Podolyák et al., Phys. Lett. B 491, 225 (2000).

[3] M. Thoennessen and B. Sherrill, Nature (London) 473, 25 (2011).

[4] I. Tanihata, Nucl. Instrum. Methods Phys. Res. B 266, 4067 (2008).

[5] L. S. Schroeder, Acta Phys. Pol. B 8, 355 (1977).

[6] S. J. Steer et al., Phys. Rev. C 84, 044313 (2011).

[7] A. Gottardo et al., Phys. Rev. Lett. 109, 162502 (2012).

[8] M. Pfützner et al., Phys. Rev. C 65, 064604 (2002).

[9] Z. Podolyák et al., Phys. Lett. B 632, 203 (2006).

[10] A. M. Denis Bacelar et al., Phys. Lett. B 723, 302 (2013).

[11] J. Kurcewicz et al., Phys. Lett. B 717, 371 (2012).

[12] M. Bowry et al., AIP Conf. Proc. 1491, 317 (2012).

[13] H. Geissel et al., Nucl. Instrum. Methods Phys. Res. B 70, 286 (1992).

[14] C. Scheidenberger et al., Nucl. Instrum. Methods Phys. Res. B 142, 441 (1998).

[15] S. Pietri et al., Nucl. Instrum. Methods Phys. Res. B 261, 1079 (2007).

[16] P. H. Regan et al., Int. J. Mod. Phys. E 17, 8 (2008).

[17] T. Kibédi et al., Nucl. Instrum. Methods Phys. Res. A 589, 202 (2008).

[18] M. Bowry, Ph.D. thesis, University of Surrey, 2013.

[19] B. Fant et al., Nucl. Phys. A 475, 338 (1987).

[20] S. K. Saha, H. Helppi, P. J. Daly, S. R. Faber, T. L. Khoo, and F. M. Bernthal, Phys. Rev. C 16, 2159 (1977).

[21] L. G. Sobotka, H. C. Griffin, and E. C. Kao, Phys. Rev. C 17, 816 (1978).

[22] F. G. Kondev, Nucl. Data Sheets 101, 521 (2004).
[23] J. Blomqvist et al., Nucl. Phys. A 554, 45 (1993).

[24] J. J. Gaimard and K. H. Schmidt, Nucl. Phys. A 531, 709 (1991).

[25] M. de Jong et al., Nucl. Phys. A 613, 435 (1997).

[26] S. A. Hjorth et al., Nucl. Phys. A 262, 328 (1976).

[27] S. C. Wang et al., Phys. Rev. C 85, 027301 (2012).

[28] C. Wheldon et al., Phys. Rev. C 74, 027303 (2006).

[29] M. G. Slocombe et al., Nucl. Phys. A 275, 166 (1977).

[30] N. Fotiades, R. O. Nelson, M. Devlin, and J. A. Becker, Phys. Rev. C 76, 014302 (2007).

[31] F. G. Kondev, Nucl. Data Sheets 105, 1 (2005).

[32] R. Broda et al., Phys. Rev. C 84, 014330 (2011).

[33] H. Hubel et al., Z. Phys. A 314, 89 (1983).

[34] F. G. Kondev and S. Lalkovski, Nucl. Data Sheets 112, 707 (2011).

[35] M. J. Martin, Nucl. Data Sheets 108, 1583 (2007).

[36] M. J. Martin, Nucl. Data Sheets 63, 723 (1991).

[37] E. Browne, Nucl. Data Sheets 99, 483 (2003).

[38] T. R. McGoram et al., Nucl. Phys. A 637, 469 (1998).

[39] E. Browne, Nucl. Data Sheets 103, 183 (2004).

[40] S. Bayer et al., Nucl. Phys. A 650, 3 (1999).

[41] G. J. Lane et al., AIP Conf. Proc. 656, 386 (2003).

[42] A. E. Stuchbery et al., Nucl. Phys. A 482, 692 (1988).

[43] G. D. Dracoulis et al., Nucl. Phys. A 467, 305 (1987).

[44] M. E. Debray et al., AIP Conf. Proc. 884, 450 (2007).

[45] A. S. Goldhaber, Phys. Lett. B 53, 306 (1974).

[46] K. H. Schmidt et al., Phys. Lett. B 300, 313 (1993).

[47] A. V. Ignatyuk, INDC Reports No. INDC(CCP)-233/L, 1985.

[48] K. A. Gladnishki et al., Phys. Rev. C 69, 024617 (2004).

[49] M. Caamaño et al., Eur. Phys. J. A 23, 201 (2005). 
[50] Z. Podolyák (private communication).

[51] S. Myalski et al., Acta Phys. Pol. B 43, 253 (2012).

[52] D. J. Decman et al., Z. Phys. A 310, 55 (1983).

[53] F. G. Kondev, Nucl. Data Sheets 109, 1527 (2008).

[54] J. M. Daugas et al., Phys. Rev. C 63, 064609 (2001).

[55] E. C. Simpson, J. A. Tostevin, Zs. Podolyák, P. H. Regan, and S. J. Steer, Phys. Rev. C 80, 064608 (2009).

[56] Z. Podolyák, Acta Phys. Pol. B 36, 1269 (2005).
[57] S. Pal and R. Palit, Phys. Lett. B 665, 164 (2008).

[58] A. Abul-Magd et al., Phys. Lett. B 60, 327 (1976).

[59] D. E. Greiner et al., Phys. Rev. Lett. 35, 152 (1975).

[60] D. J. Morrissey, Phys. Rev. C 39, 460 (1989).

[61] M. V. Ricciardi et al., Phys. Rev. Lett. 90, 212302 (2003).

[62] M. W. Reed et al., Phys. Rev. Lett. 105, 172501 (2010).

[63] M. W. Reed et al., Phys. Rev. C 86, 054321 (2012).

[64] I. Stefanescu et al., Phys. Rev. Lett. 98, 122701 (2007). 